\title{
Visitantes florales del girasol (Helianthus annuus) y de su vegetación acompañante en la Pampa Interior
}

\author{
Ana M. Chameri; Diego Medan ${ }^{122}$; Norberto H. Montaldo'; Anita I. Mantese \& \\ Mariano Devoto ${ }^{12,2}$ 四 \\ ${ }^{1}$ Universidad de Buenos Aires, Facultad de Agronomía, Cátedra de Botánica General y Cátedra de Botánica Sistemática; \\ Buenos Aires, Argentina. ${ }^{2}$ Consejo Nacional de Investigaciones Científicas y Técnicas (CONICET).
}

\begin{abstract}
Resumen. La producción, la calidad y la estabilidad de muchos cultivos, incluido el girasol, aumenta por la presencia de polinizadores que, en su mayoría, nidifican y obtienen recursos florales de los fragmentos de hábitat seminatural presentes en el paisaje. Los objetivos del estudio fueron 1) comprender la interacción del girasol con sus visitantes florales, con especial atención al efecto de la distancia al borde del cultivo, y 2) caracterizar la estructura de la red planta-polinizador, que incluye al girasol, la vegetación acompañante y sus visitantes florales, en el marco de un paisaje de la Región Pampeana sometido a uso agrícola intensivo (Gral. Villegas, provincia de Buenos Aires), donde el cultivo de girasol coexiste con la vegetación espontánea del borde. Entre los principales resultados se destaca, primero, que un grupo de abejas nativas dominó el elenco de visitantes florales; segundo, que la tasa de visita de Apis mellifera al girasol cayó hacia el interior del lote; finalmente, que los recursos florales del borde del cultivo que utilizan los polinizadores nativos son en su mayoría de origen exótico. Además, el enfoque de redes utilizado permitió ver la importancia de la vegetación circundante en el sostenimiento de las poblaciones de polinizadores del cultivo de girasol. En el presente contexto de intensificación del manejo agrícola, con la consiguiente reducción de bordes de cultivo y pérdida generalizada de biodiversidad en agroecosistemas resulta imperativo conocer mejor el valor económico y ecológico de los bordes de cultivo para sostener a los polinizadores y a la fauna benéfica en general.
\end{abstract}

[Palabras clave: polinización de cultivos, red planta-polinizador, abeja melífera, polinizador nativo, borde de cultivo]

ABSTRACT. Flower visitors of sunflower (Helianthus annuus) and its accompanying vegetation in the Inland Pampa. The yield, quality and stability of many crops, including sunflower, is increased by the presence of pollinators that mostly nest in and obtain floral resources from fragments of semi-natural habitat present in the landscape. The objectives of the study were 1) to understand the interaction of sunflower with its floral visitors, with focus on the effect of the distance to the plot margin, and 2) to characterize the structure of the plant-pollinator network that includes sunflower, the accompanying vegetation and its floral visitors, in the context of an intensively cropped landscape of the Pampean Region (Gral. Villegas, province of Buenos Aires), where the sunflower crop coexists with the spontaneous vegetation in field margins. Among the main results we highlight, first, that a group of native bees dominated the assemblage of flower visitors; second, that the visitation rate of Apis mellifera to sunflower decayed towards the center of the plot; finally, that the flower resources used by native pollinators are mostly of exotic origin. Furthermore, the network approach used allowed to visualize the importance of the surrounding vegetation in sustaining the pollinator populations of the sunflower crop. In the present context of intensification of agricultural management, with the consequent reduction of cultivation borders and generalized loss of biodiversity in agroecosystems, it is imperative to know better the economic and ecological value that field margins have in supporting pollinators and the beneficial fauna in general.

[Keywords: crop pollination, plant-pollinator network, honeybee, native pollinator, field margin]

Editor asociado: Lucas Garibaldi
Recibido: 28 de Noviembre de 2018

Aceptado: 28 de Febrero de 2020 


\section{INTRODUCCIÓN}

La transformación de los pastizales naturales que dominaban las llanuras del planeta en tierras de cultivo y pastoreo incrementó de forma sostenida la producción mundial de alimentos, pero también afectó la diversidad de especies animales y vegetales, y deterioró los servicios ecosistémicos que ellas proveen (Cardinale et al. 2012). En la Región Pampeana, la velocidad de modificación del paisaje se aceleró en décadas recientes (Paruelo et al. 2005). Una de las principales consecuencias de ese proceso ha sido la homogeneización del paisaje, lo que determina que actualmente en la Región Pampeana el componente natural de los agroecosistemas persiste sólo como una red de fragmentos (e.g., bordes de cultivo y banquinas) inmersos en una matriz de campos de cultivos o pasturas (Medan et al. 2011). La producción, la calidad y la estabilidad de muchos cultivos aumenta por la presencia de polinizadores (Klein et al. 2007; Tylianakis 2013), que en su mayoría dependen de estos fragmentos de hábitat seminaturales para nidificar y obtener recursos florales (Kremen et al. 2004). Por esto es fundamental comprender el funcionamiento de estos ambientes para realizar un manejo que conserve los servicios ecosistémicos que proveen.

El girasol es uno de los principales cultivos oleaginosos mundiales junto conla soja (Glycine max), la palma aceitera (Elaeis guineensis) y la colza (Brassica napus var. oleifera) (FAOSTAT 2016). En los últimos veinte años la producción mundial de aceite de girasol se ha más que duplicado al pasar de 7.5 millones de toneladas en 1994 a 15.8 millones de toneladas en el 2014 (FAOSTAT 2016). La Argentina es actualmente uno de los tres grandes productores mundiales (detrás de Ucrania y la Federación Rusa), y el cuarto exportador mundial de aceite de girasol (FAOSTAT 2016). En el país se siembran híbridos comerciales desde el sudeste de la Región Pampeana ( $39^{\circ}$ S) hasta la Región Chaqueña (26ㅇ S) (Trapani et al. 2003).

El rendimiento del girasol depende parcialmente de la acción de polinizadores; distintos estudios han reportado caídas de hasta ca. $50 \%$ en distintos componentes del rendimiento ante la exclusión experimental de polinizadores (Chamer 2012 y referencias incluidas). La polinización de este cultivo es efectuada principalmente por insectos y sólo en muy baja proporción por el viento (Putt 1940; Low and Pistillo 1986). La abeja melífera, abejorros, abejas silvestres y moscas son señaladas como polinizadores habituales del girasol, con gran predominio de la primera (McGregor 1976; Greenleaf and Kremen 2006). En la Argentina, la polinización del girasol fue estudiada en numerosas localidades, abarcando toda la región girasolera desde Chaco hasta el sur de Buenos Aires (Andrada et al. 2004; Hernández 2004; Torretta et al. 2009; Torretta et al. 2010; Astiz et al. 2011; Sáez et al. 2012; Cilla 2013; Chamer et al. 2015). Sin embargo, el manejo orientado a mejorar el servicio de polinización está limitado exclusivamente al aporte de colmenas de Apis mellifera (Chamer 2012; Garibaldi et al. 2014; Garibaldi et al. 2016).

En la Región Pampeana son pocos los estudios que han tenido en cuenta la vegetación de los bordes y su función en el sostén de los polinizadores del cultivo (ver, sin embargo Torreta and Poggio, 2013). Las áreas no cultivadas del paisaje, como bordes de cultivos y banquinas de caminos rurales, son reservorios de biodiversidad, incluyendo a los polinizadores nativos (Buri et al. 2014; Marshall and Moonen 2002), ya que ofrecen refugio, alimento y sitios de nidificación (Ghazoul 2005; Greenleaf and Kremen 2006). En consecuencia, el servicio de polinización que reciben los cultivos puede mejorar con la proximidad a estas áreas no cultivadas (Morandin and Winston 2006; Carvalheiro et al. 2010; De Marco and Monteiro Coelho 2004; Kremen et al. 2004; Chacoff and Aizen 2006; Klein et al. 2007). Si bien varios estudios coinciden en señalar que los polinizadores nativos muestran una disminución más abrupta en la tasa de visita a medida que aumenta la distancia a un bosque (Ricketts et al. 2004; Chacoff and Aizen 2006; Monasterolo et al. 2015) o al borde del lote (Woodcock et al. 2016), en general, estos efectos dependen de múltiples factores (e.g., estructura del paisaje, especie cultivada, tipo de vegetación dominante en los parches no cultivados, etc.).

Una herramienta útil para estudiar el sistema que forman el girasol, la vegetación del borde y sus polinizadores es el enfoque de redes ecológicas, en el que las especies son representadas por nodos unidos entre sí por nexos, que representan a las interacciones. Sin embargo, en general, el enfoque de redes ecológicas fue poco aplicado en agroecosistemas (Bohan and Woodward 2013). Por un lado, esto refleja la visión tradicional de que los sistemas seminaturales escapan al interés de la ecología convencional; y por otro 
lado, que las redes ecológicas son herramientas de utilidad práctica limitada (Memmott 2009). No obstante, recientemente se comenzaron a usar las redes de especies para comprender mejor la estructura y el funcionamiento de los agroecosistemas (Pocock et al. 2012). La descripción de las interacciones ecológicas del sistema permite identificar en los distintos niveles de la red especies o grupos de especies que poseen un papel clave para el funcionamiento del conjunto (Valladares and Salvo 1999). En el caso particular del girasol, este abordaje podría contribuir a identificar aquellas especies de plantas y polinizadores que constituyen el sostén de la función de polinización para el cultivo en particular y para el agroecosistema en general.

En este contexto, los objetivos del trabajo fueron 1) comprender la interacción del girasol con sus visitantes florales, con especial atención al efecto de la distancia al borde del cultivo, y 2) caracterizar la estructura de la red planta-polinizador que incluye al girasol, la vegetación acompañante y sus visitantes florales, en el marco de un paisaje de la Región Pampeana sometido a uso agrícola intensivo, donde el cultivo de girasol coexiste con la vegetación espontánea del borde.

\section{Materiales y MÉtodos}

Las observaciones de campo se realizaron en General Villegas, provincia de Buenos Aires, en un lote experimental perteneciente a la red de ensayos comparativos de girasol del INTA de Venado Tuerto (34 $43^{\prime} 22.6^{\prime \prime} \mathrm{S}$ $63^{\circ} 12^{\prime} 23.7^{\prime \prime} \mathrm{O} ; 127 \mathrm{~m} \mathrm{~s}$. n. m.) entre diciembre de 2002 y febrero de 2003. El diseño del ensayo consistió en dos parcelas adyacentes en las que se sembró el mismo conjunto de 34 híbridos comerciales de girasol en franjas paralelas de 5 hileras de ancho y $200 \mathrm{~m}$ de largo cada una. Las franjas se implantaron mediante siembra directa en las fechas y densidades habituales de la zona. Para este estudio se eligieron tres híbridos: Contiflor 17, Paraíso 30 y Dekasol 4050 (denominados, de aquí en más, híbridos C17, P30 y D4050, respectivamente). Los híbridos seleccionados diferían en su grado de autocompatibilidad, es decir en su capacidad de autofecundarse con éxito (C17 [excelente] > P30 [muy buena] > D4050 [media-buena], según información proporcionada por los semilleros). El lote estaba rodeado por una bordura de $\sim 10 \mathrm{~m}$ de ancho dominada por vegetación espontánea.
Serealizarondosmuestreoscomplementarios: censos estandarizados de visitantes florales en los capítulos de girasol y un muestreo no sistemático de los visitantes florales en la vegetación del borde. Los censos dentro del cultivo tuvieron 10 minutos de duración cada uno y estuvieron distribuidos entre las 9:00 y las 18:00 $\mathrm{h}$ en grupos de plantas ubicadas a 2, 20, y $100 \mathrm{~m}$ del borde del lote. Las tres distancias se censaron de forma simultánea en un mismo híbrido, y se alternó la posición de los observadores al cambiar de híbrido. Cada censo abarcó, en promedio, 4.4 capítulos (rango de 2 a 9) que presentaban al menos un tercio de sus flores abiertas. Sólo se tuvieron en cuenta los visitantes florales que contactaban las anteras o los estigmas, es decir, que fueran potenciales vectores de polen. Se realizaron cuatro censos por híbrido, distancia y parcela ( 4 censos $x 3$ híbridos $x 3$ distancias $x$ 2 parcelas), totalizando 72 censos. El muestreo en la periferia consistió en una transecta variable (variable transect walk [Westphal et al. 2008]) que abarcó todo el ancho de la bordura. Este tipo de muestreo es apropiado para ambientes en los que la distribución de recursos florales es heterogénea en el espacio; consiste en avanzar por la transecta de forma lenta, concentrando el esfuerzo de muestreo en los parches de recursos florales (Westphal et al. 2008). En los intervalos entre muestreos se coleccionaron plantas en flor y visitantes florales que luego se identificaron al menor nivel taxonómico posible.

Cuando fue necesario, los insectos se clasificaron en morfoespecies. Ciento veinte visitantes florales representativos de la mayoría de las especies se incorporaron a la colección permanentedela Cátedra deBotánica General de la Facultad de Agronomía de la Universidad de Buenos Aires. La información obtenida a campo se utilizó para construir una red planta-polinizador (Memmott 1999) que describió la ocurrencia de interacciones entre la comunidad de polinizadores, el girasol y la vegetación acompañante. Debido a que los visitantes florales de la vegetación acompañante fueron registrados en forma no sistemática, para confeccionar la red se optó por considerar sólo la información cualitativa (ocurrencia o no de cada interacción) y no cuantitativa (frecuencia de interacción). Para esto se construyó una matriz de ceros en la que cada fila representó una especie de planta y cada columna una especie de visitante floral. Luego, se les asignó un 1 a las celdas de la 
matriz que correspondieron a interacciones planta-visitante observadas en el campo.

Para describir la estructura de la red de interacciones se calculó su conectancia y su índice de anidamiento. La conectancia es una medida de la complejidad estructural de la red y representa el porcentaje de las interacciones potenciales que efectivamente ocurren (Jordano 1987). Se calcula como $\mathrm{C}=\mathrm{I} /(\mathrm{P} \times \mathrm{V}) \times 100$, donde $\mathrm{I}$ es el número de interacciones registrado (i.e., la cantidad de unos en la matriz), P es el número de especies de plantas (i.e., filas) y $\mathrm{V}$ es el número de visitantes florales (i.e., columnas). El índice de anidamiento describe en qué medida las interacciones de las especies más especialistas constituyen un subconjunto delasinteracciones de las especies más generalistas (Bascompte et al. 2003). Esta propiedad se relaciona con la robustez de la red, es decir con su capacidad de soportar la extinción progresiva de especies individuales sin que colapse su estructura y se produzcan extinciones secundarias en cascada (Bascompte and Jordano 2007). Para calcular anidamiento se utilizó el BINMATNEST (Rodríguez-Gironés and Santamaría 2006), que es muy utilizado y está implementado en R (R Core Team 2019).

Para caracterizar la variación de la tasa de visitas respecto de la identidad de los híbridos y la distancia al borde se realizaron dos análisis por separado. Por un lado, Apis mellifera; por el otro, el resto de los visitantes. Esto se debió a que $A$. mellifera fue el único visitante floral exótico y a que, a diferencia de las otras especies, su abundancia y su distribución en el paisaje pudieron verse influenciadas por la presencia de colmenas (si bien se recorrieron los caminos aledaños hasta ca. $500 \mathrm{~m}$ sin observar colmenas, no se pudo descartar por completo su presencia en las proximidades). Para cada grupo de visitantes se construyó un modelo lineal generalizado (MLG) (Bolker et al. 2009) con una distribución del error de Poisson. La variable respuesta del modelo fue el número de visitas florales del grupo correspondiente registradas por censo. Como variables explicativas se incluyeron el híbrido y la distancia al borde como variable categórica y continua, respectivamente. Por último, para caracterizar la variación en la riqueza de visitas se construyó un MLG (con error de Poisson) en el que la variable respuesta fue el número de especies de visitantes florales registrado por censo y las variables explicativas fueron el híbrido y la distancia al borde. Al haber sido realizado en un solo lote, este estudio no posee repeticiones verdaderas (i.e., otros lotes) para los tratamiento considerados (híbrido y distancia al borde), por lo que los sucesivos censos pueden ser considerados pseudorepeticiones (Hurlbert 1984), lo que limita la interpretación de los resultados.

Debido a que el número de capítulos observados en cada censo no fue constante, se incorporó a los modelos el logaritmo de esta variable como factor offset (i.e., se agregó con coeficiente 1 al predictor lineal). Un análisis preliminar reveló que el efecto de la identidad de la parcela no resultó significativo, por lo que no se incluyó como factor de bloqueo en los modelos posteriores. La estimación de los parámetros de los MLG fue realizada por cuadrados mínimos ponderados. Para determinar la significancia de los efectos de la distancia y el híbrido se compararon mediante pruebas de razón de verosimilitud (LRT, por sus siglas en inglés) modelos anidados con y sin el factor considerado. Se utilizaron para los análisis los paquetes stats (versión 3.4.1), multcomp (v. 1.4-8) y bipartite (v. 2.08) de R (R Core Team 2019).

\section{RESULTADOS}

Considerando en forma conjunta los censos en el cultivo y los muestreos en la periferia, se registraron en total 276 visitas a flores (que representaron 64 interacciones únicas entre 10 especies de plantas, incluido el girasol) y 34 especies de visitantes florales (Figura 1). Los visitantes florales fueron en su mayoría himenópteros (41.1\% de las especies), seguidos por dípteros $(23.5 \%)$, coleópteros $(20.6 \%)$ y lepidópteros (11.8\%) (Tabla S1). Las plantas acompañantes fueron en su mayoría Asteráceas (4 especies) y Brassicáceas (2 spp.) (Tabla S2). El girasol, Diplotaxis tenuifolia (Brassicáceas), Ammi majus (Apiácea) y Carduus acanthoides (Asterácea) concentraron en conjunto más del $65 \%$ de las visitas florales registradas. En particular, en los capítulos de girasol se registraron visitas de 20 especies (59\% de las especies de visitantes del sistema) repartidas en los órdenes Hymenoptera (10 especies), Diptera (3), Coleoptera (3), Lepidoptera (3) y Hemiptera (1).

Teniendo en cuenta sólo los censos sobre los capítulos de girasol, se registraron 144 visitas de diez morfotipos de visitantes distintos. El orden de visitantes dominante fue Hymenoptera y, dentro de éste, la fracción más importante en cuanto a número de especies y tasa de visitas a los capítulos fue 


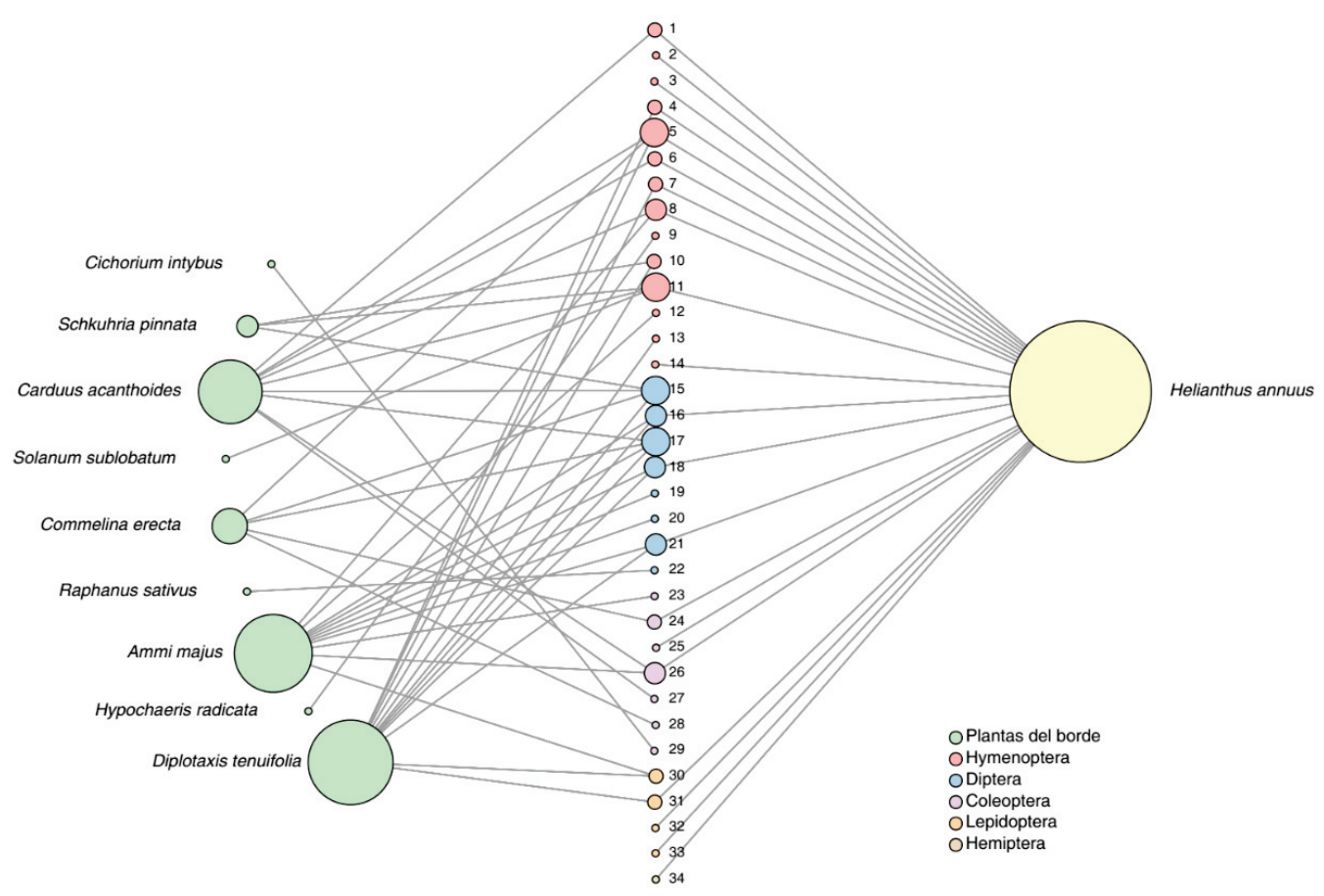

Figura 1. Red de interacciones entre plantas y visitantes florales de un lote de girasol y sus bordes en General Villegas, Buenos Aires. El tamaño de cada círculo es proporcional al grado de la especie (i.e., el número de especies del gremio opuesto con el que interactúa). Se incluyen los nombres de las plantas y los códigos de los visitantes (ver Tabla S1).

Figure 1. Network of interactions between plants (green circles) and flower visitors of a sunflower plot and its margins in General Villegas, Buenos Aires. The size of each circle represents the degree of the species (i.e., the number of species of the opposite guild with which it interacts). The name of plants and the code of flower visitors is included (see Table S1).

Apoidea (abejas) (Figura 1), grupo que incluyó a la abeja melífera y varias especies de abejas nativas de las familias Apidae, Megachilidae y Halictidae. La mayoría de estas visitas fueron realizadas por abejas nativas agrupadas en el morfotipo Melissodes (46.5\%), que incluyó cuatro especies muy difíciles de diferenciar entre sí a campo: Melissoptila tandilensis, $M$. desiderata, Melissodes tintinnans y M. rufithorax (especies 4, 5, 6 y 7, respectivamente en la Figura 1) y por la abeja doméstica Apis mellifera $(28.5 \%)$ (especie 8 en la Figura 1). Las abejas nativas observadas caminaban rápidamente por el capítulo contactando los estigmas. El análisis LRT reveló efectos significativos de la identidad del híbrido (LRT; $P=0.006$ ) (Figura 2) y de la distancia al borde (LRT; $\mathrm{P}=0.003$ ) (Figura 3) sobre la tasa de visita de A. mellifera. El híbrido D4050 fue significativamente más visitado por A. mellifera que el P30 (prueba de comparaciones múltiples de Tukey; $P=0,032$ ) y marginalmente más visitado que el C17 $(P=0.052)$; las tasas de visita a los híbridos P30 y C17 no difirieron entre sí (Figura 2). La tasa de visita de $A$. mellifera decayó a mayores

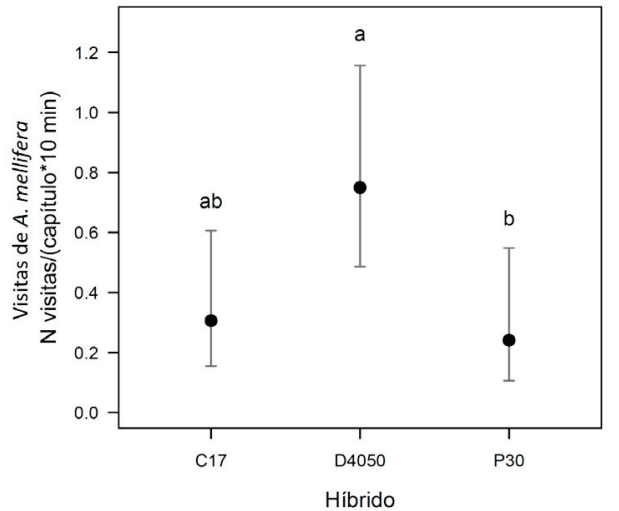

Figura 2. Efecto principal de la identidad de tres híbridos de girasol sobre la frecuencia de visitas de insectos a los capítulos en General Villegas, Buenos Aires ( $\mathrm{n}=72$ censos; se indican el valor predicho por el MLG y el intervalo de confianza del $95 \%$ ).

Figure 2. Main effect of the identity of three sunflower hybrids on insect visitation frequency to flower heads in General Villegas, Buenos Aires ( $n=72$ samples; the value predicted by the GLM and the $95 \%$ confidence interval are shown). 


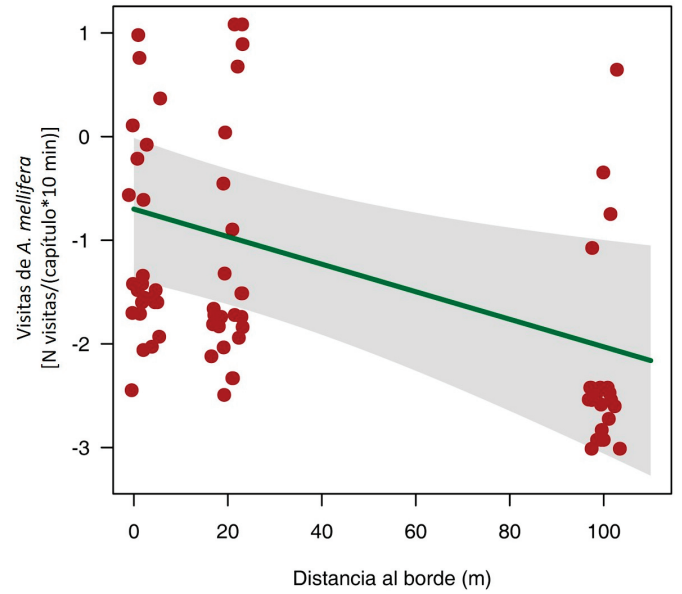

Figura 3. Efecto principal de la distancia al borde del lote sobre el número de visitas de Apis mellifera a capítulos de girasol en General Villegas, Buenos Aires ( $n=72$ censos; se indican el valor predicho por el MLG y el intervalo de confianza del 95\%). Para facilitar su interpretación visual, una pequeña cantidad de "ruido" fue agregado a la variable explicativa (debido a que muchos valores predichos eran similares); el modelo está graficado en la escala del predictor lineal.

Figure 3. Main effect of the distance to field margin on the number of visits of Apis mellifera to flower heads in General Villegas, Buenos Aires ( $\mathrm{n}=72$ samples; the value predicted by the GLM and the $95 \%$ confidence interval are shown). To aid visual interpretation a small amount of "noise" was added to the explanatory variable (as many predictions have similar values); the model is plotted on the scale of the linear predictor.

distancias del borde (GLM; $\beta=-0.013 ; Z=-2.582$; $P=0.009$ ) (Figura 3). Para el grupo formado por el resto de los visitantes florales no hubo efecto del híbrido ni de la distancia al borde sobre las tasas de visita (LRT; $P=0.23$ y $P=0,65$, respectivamente). Tampoco hubo efecto del híbrido y de la distancia sobre la riqueza de visitantes florales (LRT; $P=0.71$ y $P=0.72$, respectivamente).

La red de interacciones conformada por el girasol, sus malezas acompañantes y los visitantes florales tuvo una conectancia de 18.8\%; es decir, fue registrada sólo alrededor de la quinta parte de las interacciones potenciales. La red tuvo un índice de anidamiento 18.41 y resultó significativamente más anidada que una red ordenada al azar (prueba de t; $n=999$ redes nulas; $P<0.001)$. En esta red, las abejas nativas participaron en 23 interacciones $(35.9 \%$ de las interacciones totales), mientras que Apis mellifera estableció sólo 2 interacciones ( $3 \%$ del total).

\section{DisCUSIÓN}

En la Argentina existen escasos antecedentes de estudios que consideren el sistema formada por un cultivo, sus polinizadores y la vegetación acompañante (Chacoff and Aizen 2006; Monasterolo et al. 2015), al igual que en la Región Pampeana (ver, sin embargo, Sáez et al. 2014; Torretta and Poggio 2013). El presente trabajo pone de relieve la utilidad del enfoque de redes en el estudio de sistemas productivos. Entre los principales resultados se destaca, primero, que un grupo de abejas nativas dominó el elenco de visitantes florales; segundo, que la tasa de visita al girasol de A. mellifera evidenció una caída hacia el interior del lote y, por último, que los recursos florales del borde del cultivo que utilizan los polinizadores nativos son en su mayoría de origen exótico. En la interpretación de los resultados se debe considerar que la principal limitación del estudio es la falta de repeticiones a nivel de lote, por lo que los sucesivos muestreos en los distintos híbridos y distancias constituyen de hecho pseudorepeticiones (Hurlbert 1984). Por esto, al presente trabajo se los debe considerar un estudio de caso cuyos resultados se deberán comparar con los de estudios con repeticiones verdaderas que exploren las variaciones en el espacio y en el tiempo de un sistema cultivo-borde. A continuación, se discutirá la relevancia de los resultados y sus implicancias prácticas.

Estudios previos indican que el girasol se comporta como un cultivo cuyas flores son visitadas (y probablemente polinizadas) por un elenco diverso (Lorenzatti de Diez 1986; Torretta et al. 2010). En coincidencia con esto, el elenco de visitantes de este estudio incluyó 20 especies de 4 órdenes de insectos. Las especies de Apoidea capturadas coinciden en gran parte con las registradas en dos zonas cercanas al sitio de estudio (Departamento de General Roca en Córdoba y Partido de Carlos Casares en Buenos Aires) (Torretta et al. 2010).

En la Argentina se reportaron visitas al girasol de abejas de distintas familias de Apoidea (Halictidae, Andrenidae, Megachilidae, y Apidae), coleópteros, dípteros y lepidópteros (Sívori 1941; Lorenzatti de Diez 1986; Torretta et al. 2010). Si bien la mayoría de los estudios mencionan a $A$. mellifera como la abeja que visita con mayor frecuencia al girasol (e.g., en Torretta et al. 2010, esta especie realizó 
el 93\% de las visitas registradas), el grupo dominante en el presente estudio fue el de las abejas nativas de la tribu Eucerini (especies de los géneros Melissodes y Melissoptila) (Figura 1 y Tabla S1). Esta frecuencia elevada de abejas nativas se podría deber a que el lote de girasol y sus bordes ofrecen recursos florales abundantes y un sustrato apto para su nidificación (incidentalmente se observaron nidos de $M$. tintinnans y $M$. rufithorax tanto en el interior como en el borde del lote estudiado). Esto coincide con un relevamiento sistemático de nidos y de abejas en la región que sugiere que los sitios seleccionados por Melissodes para nidificar varían de año a año en función de la cercanía a recursos florales abundantes (Cilla 2013). En un estudio realizado en Carlos Casares y General Villegas (Cilla 2013) el polen de $H$. annuus representó un componente importante de la dieta de $M$. tintinnans (41\% del polen de las cargas escopales de 247 hembras) y $M$. rufithorax $(30 \%$ del polen de las cargas escopales de 147 hembras). Esta evidencia sugiere que Melissodes tintinnans y M. rufithorax (en especial la primera) deben ser consideradas especies potencialmente importantes en la polinización del cultivo (Cilla 2013). Esto concuerda con que en América del Norte al girasol lo visitan muchas especies de eucerinos, posiblemente porque en esa región el género Helianthus y este grupo de abejas comparten una historia evolutiva común (Hurd et al. 1980; Parker et al. 1981).

La baja frecuencia relativa de visitas de abejas melíferas encontrada podría estar asociada a la ausencia de colmenas comerciales en las cercanías o a la presencia de recursos florales alternativos más apetecidos por esta especie. En este sentido, Basualdo et al. (2000) reportaron una baja preferencia de la abeja melífera por el girasol cuando florecen simultáneamente otras especies más apetecidas (e.g., Carduus acanthoides visitada por A. mellifera en Gral. Villegas). En el presente estudio se registraron varias especies vegetales que resultan fuentes habituales de polen y néctar para Apis (Diplotaxis tenuifolia, Schkuhria pinnata, Hypochaeris radicata, Ammi majus y Carduus acanthoides). Esta competencia por polinizadores entre el cultivo y el borde también podría explicar la caída en la tasa de visitas de $A$. mellifera hacia el interior del lote, lo que se observó en lotes de girasol en otras localidades de la Región Pampeana (Torretta 2007; Sáez et al. 2014) y en otros sistemas borde-cultivo (Mitchell et al. 2009; Nicholls and Altieri 2013). En el mismo sentido, un estudio del rendimiento de los híbridos de este lote (Chamer 2012) reveló que el híbrido D4050 (el menos autocompatible) tuvo una mayor tasa de fructificación y contenido total de aceite por capítulo a $2 \mathrm{~m}$ de distancia del borde que a $100 \mathrm{~m}$. Esto sugiere que, en el caso de la polinización por $A$. mellifera, el tamaño y la forma del lote, y la composición de la comunidad de plantas del borde pueden tener un efecto sobre la calidad del servicio de polinización que recibe el cultivo y sobre el rendimiento resultante.

Otro aspecto interesante es la preferencia que mostró $A$. mellifera por un determinado híbrido de girasol. Un comportamiento similar fue reportado en lotes de producción de semilla híbrida donde el cuajado de frutos puede resultar afectado si las líneas parentales ejercen distintos grados de atracción hacia las abejas visitantes. La evidencia indica que la preferencia de las abejas depende de las diferencias entre híbridos en el patrón de reflectancia en el espectro ultravioleta de las flores liguladas (Charlet et al. 1997), la proporción de sacarosa en el néctar (PhamDelegue et al. 1990) y la estructura de los nectarios (Sammataro et al. 1985). Además, es probable que haya diferencias entre híbridos en el largo del tubo floral (Sammataro et al. 1983) y que las preferencias de $A$. mellifera se deban a la proporción de néctar que es capaz de extraer de las flores (du Toit and Coetzer 1991; Portlas et al. 2018). Los resultados alientan a realizar una descripción detallada de las diferencias entre híbridos en las características florales ya que puede resultar provechoso orientar el mejoramiento del cultivo a incrementar su atractivo floral.

El análisis de las interacciones reveló que, como es común en sistemas plantapolinizador, la red posee un patrón fuertemente anidado. Esto implica que la comunidad es dominada por un núcleo de especies generalistas de plantas (H. annuus, $D$. tenuifolia, $A$. majus, C. acanthoides) y visitantes florales (M. rufithorax, Toxomerus sp., Allograpta sp. y Augochloropsis sp.) que interactúan con especies más especialistas del gremio opuesto. Si bien es probable que la dominancia de $H$. annuus en el sistema se deba, en parte, a que el esfuerzo de muestreo de polinizadores fue mayor dentro de los lotes que en la vegetación de la periferia, su rol central en la red de interacciones refleja también su importancia como fuente de recursos en forma de néctar y polen para los insectos. Es destacable que, si 
bien A. mellifera estaba presente en el sistema, no ocupó una posición dominante en la red, sobre todo teniendo en cuenta el fuerte efecto negativo que puede generar en la red plantapolinizador local al establecerse (Valido et al. 2019). En ese sentido, el sistema estudiado parece presentar un nivel de degradación bajo.

El enfoque de redes utilizado permitió identificar especies de visitantes florales que actuaron como conectores entre la vegetación del borde y el cultivo al visitar flores en ambos hábitats (Tabla S1). Las dos especies más relevantes en esta función fueron Melissodes rufithorax (\#5 en la Figura 1) y Augochloropsis sp. (\#11 en la Figura 1), que además están entre las especies más generalistas del sistema. Este enfoque permitió además visualizar la importancia de la vegetación circundante en el sostenimiento de las poblaciones de polinizadores del cultivo de girasol. Siete de las nueve malezas fueron visitadas por himenópteros, el grupo más relevante en la polinización del girasol. En este sentido, la vegetación de los bordes del cultivo parece ser un elemento fundamental del paisaje al contribuir, por un mecanismo de derrame (spillover) de polinizadores (Woodcock et al. 2016), a garantizar el servicio de polinización que recibe el cultivo. Este mismo efecto fue descripto para otros cultivos y sistemas productivos (Blaauw and Isaacs 2014a; Blaauw and Isaacs 2014b; Garibaldi et al. 2014; Feltham et al. 2015). Por ejemplo, en Sudáfrica, la cercanía de cultivos de girasol a áreas de hábitat natural incrementó la diversidad de polinizadores que visitaban el cultivo y la permanencia de estos en el cultivo fue mayor cuando hubo una alta proporción de malezas dentro del mismo, coincidiendo ambas situaciones con una alta productividad del cultivo (Carvalheiro et al. 2011).

Una importante ventaja del manejo de abejas nativas como polinizadores de cultivos es evitar el impacto ecológico causado por la introducción de abejas exóticas (Aizen et al. 2008; Vergara and Badano 2009). En este sentido, este trabajo sugiere que Melissodes tintinnans y $M$. rufithorax (en especial la primera) pueden ser especies importantes polinizadoras del cultivo y por lo tanto ameritan estudios más profundos. Conocer en detalle su ciclo de vida, con especial atención al hábitat de nidificación y al recurso polínico que emplean para alimentar sus larvas, complementaría de manera ventajosa los estudios de este tipo disponibles en la actualidad en agroecosistemas de la Argentina (Cilla [2013] para algunas especies de Melissodes; Torretta and Poggio [2013] para Megachile gomphrenoides).

Estudios previos de la polinización del girasol pusieron el acento en describir el elenco de visitantes del cultivo (Torretta et al. 2009; Torretta et al. 2010) o en la influencia de las características del borde en su elenco de visitantes (Sáez et al. 2014). En este contexto, el empleo de redes resulta un enfoque unificador ya que permite comprender cómo la flora del borde se conecta con el cultivo por intermedio de los insectos que vistan a ambos. En el presente contexto de intensificación del manejo agrícola, con la consiguiente reducción de bordes de cultivo y pérdida generalizada de biodiversidad en agroecosistemas, es imperativo conocer mejor el valor económico y ecológico que poseen los bordes de cultivo para el sostenimiento de los polinizadores, y de la fauna benéfica en general (Garibaldi et al. 2011).

Agradecimientos. Agradecemos al Instituto Nacional de Tecnología Agropecuaria de Venado Tuerto por otorgar el permiso para realizar el presente estudio en el lote experimental, y a Arturo Roig Alsina (Museo Argentino de Ciencias Naturales "Bernardino Rivadavia") por su ayuda en la identificación de los insectos. Este trabajo fue financiado por un subsidio PICT de la Agencia Nacional de Promoción Científica y Tecnológica otorgado a Diego Medan.

\section{REFERENCIAS}

Aizen, M. A., C. L. Morales, and J. M. Morales. 2008. Invasive mutualists erode native pollination webs. PLoS Biology 6(2):e31. https://doi.org/10.1371/journal.pbio.0060031.

Andrada, A., A. Valle, P. Paoloni, and L. Gallez. 2004. Pollen and nectar sources used by honeybee colonies pollinating sunflower (Helianthus annuus) in the Colorado River Valley, Argentina. Boletín de la Sociedad Argentina de Botánica 39:75-82.

Astiz, V., L. Iriarte, A. Flemmer, and L. Hernández. 2011. Self-compatibility in modern hybrids of sunflower (Helianthus annuus L.) fruit set in open and self-pollinated (bag isolated) plants grown in two different locations. Helia 34:129138. https://doi.org/10.2298/HEL1154129A.

Bascompte, J., and P. Jordano. 2007. Plant-animal mutualistic networks: The architecture of biodiversity. Annual Review 
of Ecology, Evolution and Systematics 38:567-593. https://doi.org/10.1146/annurev.ecolsys.38.091206.095818.

Bascompte, J., P. Jordano, C. Melián, and J. M. Olesen. 2003. The nested assembly of plant-animal mutualistic networks. Proceedings of the National Academy of Sciences 100:9383-9387. https:/ / doi.org/10.1073/pnas.1633576100.

Basualdo, M., E. Bedascarrasbure, and D. De Jong. 2000. Africanized honey bees (Hymenoptera: Apidae) have a greater fidelity to sunflowers than european bees. Journal of Economic Entomology 93:304-307. https://doi.org/10.1603/ 0022-0493-93.2.304.

Blaauw, B. R., and R. Isaacs. 2014a. Flower plantings increase wild bee abundance and the pollination services provided to a pollination-dependent crop. Journal of Applied Ecology 51:890-898. https://doi.org/10.1111/1365-2664.12257.

Blaauw, B. R., and R. Isaacs. 2014b. Larger patches of diverse floral resources increase insect pollinator density, diversity and their pollination of native wildflowers. Basic and Applied Ecology 15:701-711. https://doi.org/ 10.1016/j.baae.2014.10.001.

Bohan, D. A., and G. Woodward. 2013. Preface: Editorial Commentary: The Potential for Network Approaches to Improve Knowledge, Understanding and Prediction of the Structure and Functioning of Agricultural Systems. Pp. xiii-xviii in W. Guy and A. B. David (eds.). Advances in Ecological Research. Academic Press. https://doi.org/ 10.1016/B978-0-12-420002-9.10000-9.

Bolker, B., M. Brooks, C. Clark, S. Geange, J. Poulsen, H. Stevens, and J.-S. White. 2009. Generalized linear mixed models: a practical guide for ecology and evolution. Trends in Ecology and Evolution 24:127-135. https://doi.org/ 10.1016/j.tree.2008.10.008.

Buri, P., J.-Y. Humbert, and R. L. Arlettaz. 2014. Promoting pollinating insects in intensive agricultural matrices: fieldscale experimental manipulation of hay-meadow mowing regimes and its effects on bees. PloS One 9:e85635-e85635. https://doi.org/10.1371/journal.pone.0085635.

Cardinale, B. J., J. E. Duffy, A. González, D. U. Hooper, C. Perrings, P. Venail, A. Narwani, G. M. Mace, D. Tilman, D. A. Wardle, A. P. Kinzig, G. C. Daily, M. Loreau, J. B. Grace, A. Larigauderie, D. S. Srivastava, and S. Naeem. 2012. Biodiversity loss and its impact on humanity. Nature 486:59-67. https:/ / doi.org/10.1038/nature11148.

Carvalheiro, L. G., C. L. Seymour, R. Veldtman, and S. W. Nicolson. 2010. Pollination services decline with distance from natural habitat even in biodiversity-rich areas. Journal of Applied Ecology 47:810-820. https:/ / doi.org/10.1111/ j.1365-2664.2010.01829.x.

Carvalheiro, L. G., R. Veldtman, A. G. Shenkute, G. B. Tesfay, C. W. W. Pirk, J. S. Donaldson, and S. W. Nicolson. 2011. Natural and within-farmland biodiversity enhances crop productivity. Ecology Letters 14:251-259. https://doi.org/ 10.1111/j.1461-0248.2010.01579.x.

Cilla, G. 2013. Biología de abejas nativas de la tribu Eucerini (Hymenoptera, Apidae): uso de los recursos polínicos en la región pampeana argentina. Tesis doctoral. Universidad de Buenos Aires. Buenos Aires. Pp. xvi + 399.

Chacoff,N.P., and M. A. Aizen. 2006. Edge effects on flower-visiting insects in grapefruit plantations bordering premontane subtropical forest. Journal of Applied Ecology 43:18-27. https:/ / doi.org/10.1111/j.1365-2664.2005.01116.x.

Chamer, A. M. 2012. Influencia del servicio de polinización sobre el rendimiento del girasol (Helianthus annuus L.) en la Argentina. Tesis doctoral. Universidad de Buenos Aires. Buenos Aires. Pp. 121.

Chamer, A. M., D. Medan, A. Mantese, and N. Bartoloni. 2015. Impact of pollination on sunflower yield: Is pollen amount or pollen quality what matters? Field Crops Research 176:61-70. https://doi.org/10.1016/j.fcr.2015.02.001.

Charlet, L. D., G. L. Brewer, and B. A. Franzmann. 1997. Sunflower insects. Pp. 245-261 in A. A. Schneiter (ed.). Sunflower Technology and Production. ASA, CSSA and SSSA Madison, Wisconsin, USA.

De Marco, P., and F. Monteiro Coelho. 2004. Services performed by the ecosystem: forest remnants influence agricultural cultures' pollination and production. Biodiversity and Conservation 13:1245-1255. https://doi.org/10.1023/B: BIOC.0000019402.51193.e8.

du Toit, A. P., and L. A. Coetzer. 1991. Difference in potential attractiveness to bees of seventeen South African sunflower (Helianthus annuus) cultivars. International Society for Horticultural Science (ISHS), Leuven, Belgium. Pp. 288-293. https://doi.org/10.17660/ActaHortic.1991.288.45.

FAOSTAT. 2016. Food and Agriculture Organization of the United Nations Statistics Database. URL: www.fao.org/ faostat/es Last accessed 14/11/2018.

Feltham, H., K. Park, J. Minderman, and D. Goulson. 2015. Experimental evidence that wildflower strips increase pollinator visits to crops. Ecology and Evolution 5:3523-3530. https://doi.org/10.1002/ece3.1444.

Garibaldi, L. A., M. A. Aizen, A. M. Klein, S. A. Cunningham, and L. D. Harder. 2011. Global growth and stability in agricultural yield decrease with pollinator dependence. Proceedings of the National Academy of Sciences 108:59095914. https://doi.org/10.1073/pnas.1012431108.

Garibaldi, L. A., L. G. Carvalheiro, S. D. Leonhardt, M. A. Aizen, B. R. Blaauw, R. Isaacs, M. Kuhlman, D. Kleijn, A. M. Klein, C. Kremen, L. Morandin, J. A. Scheper, and R. Winfree. 2014. From research to action: enhancing crop yield through wild pollinators. Frontiers in Ecology and the Environment 12:439-447. https://doi.org/10.1890/130330.

Garibaldi, L. A., L. G. Carvalheiro, B. E. Vaissière, B. Gemmill-Herren, J. Hipólito, B. M. Freitas, H. T. Ngo, N. Azzu, A. Sáez, J. Åström, J. An, B. Blochtein, D. Buchori, F. J. Chamorro García, F. Oliveira da Silva, K. Devkota, M. de Fátima Ribeiro, L. Freitas, M. C. Gaglianone, M. Goss, M. Irshad, M. Kasina, A. J. S. Pacheco Filho, L. H. Piedade Kiill, P. Kwapong, G. Nates Parra, C. Pires, V. Pires, R. S. Rawal, A. Rizali, A. M. Saraiva, R. Veldtman, B. F. Viana, S. Witter, and H. Zhang. 2016. Mutually beneficial pollinator diversity and crop yield outcomes in small and large farms. Science 351:388-391. https:/ / doi.org/10.1126/science.aac7287.

Ghazoul, J. 2005. Buzziness as usual? Questioning the global pollination crisis. Trends in Ecology and Evolution 20: 367-373. https://doi.org/10.1016/j.tree.2005.09.006. https:/ /doi.org/10.1016/j.tree.2005.04.026. 
Greenleaf, S. S., and C. Kremen. 2006. Wild bees enhance honey bees' pollination of hybrid sunflower. Proceedings of the National Academy of Sciences 103:13890-13895. https://doi.org/10.1073/pnas.0600929103.

Hernández, L. F. 2004. Visit path pattern of the honeybee (Apis mellifera L.) on the sunflower capitulum correspondence with the location of seedless and incompletely developed fruits. Helia 31:1-16. https://doi.org/ 10.2298/HEL0848001H.

Hurlbert, S. H. 1984. Pseudoreplication and the design of ecological field experiments. Ecological Monographs 54: 187-211. https://doi.org/10.2307/1942661.

Hurd, P. D. Jr., W. E. LeBerge, and L. E. Gorton. 1980. Principal sunflower bees of North America with emphasis on the southeastern United States (Hymenoptera: Apoidea). Smithsonian Contributions to Zoology 310:1-157. https: //doi.org/10.5479/si.00810282.310.

Jordano, P. 1987. Patterns of mutualistic interactions in pollination and seed dispersal: connectance, dependence, asymmetries and coevolution. The American Naturalist 129:657-677. https://doi.org/10.1086/284665.

Klein, A.-M., B. E. Vaissière, J. H. Cane, I. Steffan-Dewenter, S. A. Cunningham, C. Kremen, and T. Tscharntke. 2007. Importance of pollinators in changing landscapes for world crops. Proceedings of the Royal Society Biological Sciences Series B 274:303-313. https://doi.org/10.1098/rspb.2006.3721.

Kremen, C., N. M. Williams, R. L. Bugg, J. P. Fay, and R. W. Thorp. 2004. The area requirements of an ecosystem service: crop pollination by native bee communities in California. Ecology Letters 7:1109-1119. https://doi.org/10.1111/j.14610248.2004.00662.x.

Lorenzatti de Diez, S. 1986. Estudios de polinización de girasol (Helianthus annuus L.) por abejas melíferas (Apis mellifera L.). Oleico 33:41-50.

Low, A., and G. Pistillo.1986. The self-fertility status of some sunflower cultivars in Australia. Field Crops Research 14:233-245. https://doi.org/10.1016/0378-4290(86)90061-4.

Marshall, E. J. P., and A. C. Moonen. 2002. Field margins in northern Europe: their functions and interactions with agriculture. Agriculture, Ecosystems and Environment 89:5-21. https://doi.org/10.1016/S0167-8809(01)00315-2.

McGregor, S. E. 1976. Insect pollination of cultivated crop plants. Agric. Res. Ser. USDA, Washington, D.C.

Medan, D., J. P. Torretta, K. Hodara, E. B. de la Fuente, and N. H. Montaldo. 2011. Effects of agriculture expansion and intensification on vertebrate and invertebrate diversity in the Pampas of Argentina. Biodiversity and Conservation 20:3077-3100. https://doi.org/10.1007/s10531-011-0118-9.

Memmott, J. 1999. The structure of a plant-pollinator food web. Ecology Letters 2:276-280. https://doi.org/10.1046/ j.1461-0248.1999.00087.x.

Memmott, J. 2009. Food webs: a ladder for picking strawberries or a practical tool for practical problems? Philosophical Transactions of the Royal Society B: Biological Sciences 364:1693-1699. https://doi.org/10.1098/rstb.2008.0255.

Mitchell, R. J., R. J. Flanagan, B. J. Brown, N. M. Waser, and J. D. Karron. 2009. New frontiers in competition for pollination. Annals of Botany 103:1403-1413. https://doi.org/10.1093/aob/mcp062.

Monasterolo, M., M. L. Musicante, G. R. Valladares, and A. Salvo. 2015. Soybean crops may benefit from forest pollinators. Agriculture, Ecosystems and Environment 202:217-222. https://doi.org/10.1016/j.agee.2015.01.012.

Morandin, L. A., and M. L. Winston. 2006. Pollinators provide economic incentive to preserve natural land in agroecosystems. Agriculture, Ecosystems and Environment 116:289-292. https://doi.org/10.1016/j.agee.2006.02.012.

Nicholls, C. I., and M. A. Altieri. 2013. Plant biodiversity enhances bees and other insect pollinators in agroecosystems. A review. Agronomy for Sustainable Development 33:257-274. https://doi.org/10.1007/s13593-012-0092-y.

Parker, F., V. Tepedino, and G. Bohart. 1981. Notes on the biology of a common sunflower bee, Melissodes (Eumelissodes) agilis Cresson. Journal of the New York Entomological Society 89:43-52.

Paruelo, J. M., Guerschman, J. P., and Verón, S. R. 2005. Expansión agrícola y cambios en el uso del suelo. Ciencia Hoy 15:14-23.

Pham-Delegue, M. H., P. Etievant, E. Guichard, R. Marilleau, P. Douault, J. Chauffaille, and C. Masson. 1990. Chemicals involved in honeybee-sunflower relationship. Journal of Chemical Ecology 16:3053-3065. https://doi.org/10.1007/ BF00979612.

Pocock, M. J. O., D. M. Evans, and J. Memmott. 2012. The robustness and restoration of a network of ecological networks. Science 335:973-977. https://doi.org/10.1126/science.1214915.

Portlas, Z. M., J. R. Tetlie, D. Prischmann-Voldseth, B. S. Hulke, and J. R. Prasifka. 2018. Variation in floret size explains differences in wild bee visitation to cultivated sunflowers. Plant Genetic Resources: Characterization and Utilization 16:498-503. https://doi.org/10.1017/S1479262118000072.

Putt, E. D. 1940. Observations on morphological characters and flowering processes in the sunflower (Helianthus annuus L.). Scientific Agriculture 21:167-179.

R Core Team. 2019. R: A language and environment for statistical computing. R Foundation for Statistical Computing, Vienna, Austria. URL: www.R-project.org.

Ricketts, T. H., G. C. Daily, P. R. Ehrlich, and C. D. Michener. 2004. Economic value of tropical forest to coffee production. Proceedings of the National Academy of Sciences 101:12579-12582. https://doi.org/10.1073/pnas.0405147101.

Rodríguez-Gironés, M. A., and L. Santamaría. 2006. A new algorithm to calculate the nestedness temperature of presence-absence matrices. Journal of Biogeography 33:924-935. https://doi.org/10.1111/j.1365-2699.2006.01444.x.

Sáez, A., M. Sabatino, and M. A. Aizen. 2012. Interactive Effects of Large- and Small-Scale Sources of Feral Honey-Bees for Sunflower in the Argentine Pampas. Plos One 7:e30968. https://doi.org/10.1371/journal.pone.0030968. 
Sáez, A., M. Sabatino, and M. A. Aizen. 2014. La diversidad floral del borde afecta la riqueza y abundancia de visitantes florales nativos en cultivos de girasol. Ecología Austral 24:94-102.

Sammataro, D., E. H. Erickson, and M. B. Garment. 1983. Intervarietal structural differences of sunflower (Helianthus annuus) florets their importance to honey bee visitation. In: Proc. 5th Sunflower Res. Workshop. Natl. Sunflower Assoc., Minot, ND.

Sammataro, D., E. H. Erickson, and M. B. Garment. 1985. Ultrastructure of the sunflower nectary. Journal of Apicultural Research 24:150-160. https://doi.org/10.1080/00218839.1985.11100665.

Sívori, E. M. 1941. Biología floral del girasol. Revista Argentina de Agronomía 8:150-154.

Torretta, J. P. 2007. Entomofauna asociada a la polinización del girasol (Helianthus annuus L.) en Argentina. Facultad de Ciencias Exactas y Naturales.

Torretta, J. P., D. Medan, A. Roig-Alsina, and N. H. Montaldo. 2010. Visitantes florales diurnos del girasol (Helianthus annuus, Asterales: Asteraceae) en la Argentina. Revista de la Sociedad Entomológica Argentina 69:17-32.

Torretta, J. P., F. Navarro, and D. Medan. 2009. Visitantes florales nocturnos del girasol (Helianthus annuus, Asterales: Asteraceae) en la Argentina. Revista de la Sociedad Entomológica Argentina 68:339-350.

Torretta, J. P., and S. L. Poggio. 2013. Species diversity of entomophilous plants and flower-visiting insects is sustained in the field margins of sunflower crops. Journal of Natural History 47:139-165. https://doi.org/10.1080/ 00222933.2012.742162.

Trapani, N., M. López-Pereira, V. O. Sadras, and A. J. Hall. 2003. Ciclo ontogénico, dinámica del desarrollo y generación del rendimiento y la calidad en girasol. Pp. 203-241 in A. J. Pascale (ed.). Producción de granos: Bases funcionales para su manejo. Editorial Facultad de Agronomía Buenos Aires, Argentina.

Tylianakis, J. M. 2013. The global plight of pollinators. Science 339:1532-1533. https://doi.org/10.1126/ science.1235464.

Valido, A., M. C. Rodríguez-Rodríguez, and P. Jordano. 2019. Honeybees disrupt the structure and functionality of plant-pollinator networks. Scientific Reports 9:4711. https://doi.org/10.1038/s41598-019-41271-5.

Valladares, G. R., and A. Salvo. 1999. Insect-plant food webs could provide new clues for pest management. Environmental Entomology 28:539-544. https://doi.org/10.1093/ee/28.4.539.

Vergara, C. H., and E. I. Badano. 2009. Pollinator diversity increases fruit production in Mexican coffee plantations: The importance of rustic management systems. Agriculture, Ecosystems and Environment 129:117-123. https://doi.org/ 10.1016/j.agee.2008.08.001.

Westphal, C., R. Bommarco, G. Carré, E. Lamborn, N. Morison, T. Petanidou, S. G. Potts, S. P. M. Roberts, H. Szentgyörgyi, T. Tscheulin, B. E. Vaissière, M. Woyciechowski, J. C. Biesmeijer, W. E. Kunin, J. Settele, I. Steffan-Dewenter. 2008. Measuring bee diversity in different European habitats and biogeographical regions. Ecological Monographs 78: 653-671. https://doi.org/10.1890/07-1292.1.

Woodcock, B. A., J. M. Bullock, M. McCracken, R. E. Chapman, S. L. Ball, M. E. Edwards, M. Nowakowski, and R. F. Pywell. 2016. Spill-over of pest control and pollination services into arable crops. Agriculture, Ecosystems and Environment 231:15-23. https://doi.org/10.1016/j.agee.2016.06.023. 